\title{
La innovación empresarial en la Pyme, desde la integración regional en América Latina
}

Fecha de recepción: 29 de marzo de 2011

Fecha de aceptación: 30 de junio de 2011

\author{
Ángela Julieta Mora Ramírez \\ amoraram@poli.edu.co \\ Politécnico Grancolombiano
}

Economista de la Universidad Pedagógica y Tecnológica (Tunja), especialista en Integración en el Sistema internacional de la Universidad Javeriana, es investigadora y docente desde hace más de diez años. Fundadora de la revista electrónica Expectativas de la Universidad Jorge Tadeo Lozano, editora y gestora de revistas web, escribe para revistas de divulgación e indexadas, nacionales e internacionales, sobre temas pedagógicos y de su área específica de conocimiento: la integración regional económica.

Es árbitro y par de revistas indexadas nacionales e internacionales. Actualmente está terminando la maestría en Educación con Énfasis en Investigación y es Directora del Centro de Investigación "Colombia Regional y Empresarial" CICRE del Politécnico Grancolombiano y directora de Investigación Formativa de la Institución.

\section{Resumen}

Este artículo de revisión es un acercamiento descriptivo de los procesos de innovación de las empresas Pymes desde los acuerdos de integración regional suramericanos, caso específico Colombia actualmente.

El proceso de globalización requiere de experiencias nuevas en las empresas con fines de expansión y articulación internacional, la esencia de los procesos de negociación requiere de una visión amplia sobre la innovación en la Pyme.

Esta innovación permite, desde la revisión documental, establecer la necesidad de aclarar este concepto, sin perder la esencia de la Pyme en el entorno mundial, más ahora donde la ventaja comparativa es mayor. Además permite establecer escenarios nuevos de generación de productividad y competitividad de estos pequeños mercados productivos y generadores de ventajas sobre la indus-

\section{Abstract}

This review is a descriptive approach of the innovation processes of SMEs business, from the South American regional integration agreements, being Colombia nowadays the specific case.

The process of globalization requires new experiences in companies that search for international expansion and articulation. The essence of negotiation processes requires a broad vision about innovation in SMEs.

This innovation allows establishing the need to clarify this concept from literature, without losing the essence of the SMEs in the global environment. Now that the comparative advantage is greater, it establishes new scenarios of generation, productivity, and competitiveness within these small productive markets in order to generate advantages in the national industry. This will really help other countries to produce, connect, and check data 
tria nacional, que aporte realmente a la producción de los países. Finalmente permite vincular y revisar datos sobre innovación en las Pymes, desde su aporte nacional a los contextos de globalización e interdependencia.

\section{Palabras clave}

Integración, innovación, Pymes, Colombia. about innovation in SMEs from their national contribution to the context of globalization, and interdependence.

\section{Keywords}

Integration, Innovation, SMEs, Colombia. 


\section{Introducción}

Según la página Oficial de la Comunidad Andina (2009):

El proceso de globalización somete a las economías andinas a un conjunto de retos, lo que obliga a una revisión de las estrategias de desarrollo nacional, con el fin de superar la dependencia de tecnologias generadas en otros contextos, con la consiguiente pérdida de mercados por parte de nuestras empresas, aún en los mercados tradicionales. Esta situación es producto de las diferencias en las capacidades de generación de conocimiento entre nuestros paises y paises desarrollados y su eventual transformación de innovaciones que contribuyan a elevar el valor agregado de los bienes y servicios producidos por nuestras empresas.

Los países, sus políticas y sus economías son cambiantes y establecen nuevas formas de dinámica que permean las instituciones, haciéndolas ágiles y altamente competitivas para el apoyo y el incentivo a las pequeñas y medianas empresas de la subregión latinoamericana, especialmente para los países andinos.

Por tanto, el objetivo de este artículo es desarrollar de manera descriptiva una revisión documental, incluyendo un análisis de fuente secundaria, al mismo tiempo que una proyección de las opciones de la política de integración en Pymes sobre innovación, dirigido a la internacionalización de estas.

\section{La innovación en los países andinos}

Según la página oficial de la CAN (2009):

En los actuales momentos, debido a los diversos y simultáneos procesos de negociaciones comerciales en marcha, en los que se aspira hacerlo favorablemente, intentando obtener mayores ventajas posibles frente a terceros, las pequeñas y medianas empresas corren el peligro de ser severamente afectadas por falta de capacidades competitivas, con los consiguientes problemas de destrucción del tramado productivo y la pérdida de empleo. La mejora de dichas capacidades competitivas exige un proceso de mejoramiento continuo de la calidad de los productos desarrollados y ese mejoramiento tiene que ir acompañado de incorporación progresiva de innovaciones, tanto a nivel de los productos como de procesos. Podemos por lo tanto, aseverar que un desarrollo económico sostenido está fuertemente condicionado por la capacidad de incorporar conocimientos e innovación tecnológica a los sistemas productivos, como factor estratégico de competitividad.

Este proceso de competencia implica acercamientos de la empresa Pyme sobre la política exterior común, inmersa en el tratado y en el desarrollo productivo de estas empresas en el mercado mundial.

Continúo citando la CAN (2009):

Es indudable entonces, que la apertura de los mercados impone la necesidad de innovar, adoptar, adaptar y asimilar tecnología, como 
via para enfrentar los retos que impone la competencia de los mercados. Para ello, es necesario contar con un sistema de innovación que integre en ese esfuerzo a todos los actores vinculados al proceso de desarrollo del sector productivo y esto no sólo se refiere a la vinculación entre los centros generadores de nuevos conocimientos y tecnologías, sino también a los usuarios del sector empresarial y más aún, a la sociedad en general como copartícipe, demandante y consumidor final de los productos y servicios a su disposición. Para la consolidación de un sistema de innovación que contribuya al mejoramiento de la competitividad, es necesario contar con una importante capacidad de investigación y desarrollo (I y D) y un sector empresarial con disposición a asumir los retos de la innovación, colaborando con las actividades de I y D.

Esto genera un proceso de transferencia tecnológica dinámico que permita procesos de interdependencia y multilateralismo que aporten y vinculen estrategias de productividad enfocada a la innovación, el desarrollo del aparato productivo y la especificación y diversificación de la mano de obra.

Especializando los mercados con productos y con la generación de nuevos conocimientos que repercuta en la productividad y eficiencia de los países de la subregión.

La integración se ha preocupado desde su esencia por la cooperación y reciprocidad desde su estructura económica hasta su estructura política-social, de manera vinculante y con miras de conformar crecimiento y desarrollo armónico.
Con la especificación de la innovación desde el aparato productivo de las empresas y en especial de las Pymes, a sabiendas que la mayoría de estas generan la dinámica potencial en innovación que puede generar un proceso de desarrollo económico creciente.

Según la página oficial de la CAN (2009):

Dado que las exportaciones de la región están constituidas fundamentalmente por productos primarios, con poco valor agregado y sometidos a los vaivenes de la demanda y a la erraticidad de los precios, debe hacerse un esfuerzo para darle un valor agregado, basado en la incorporación de innovaciones, mejoramiento de la calidad y mayor eficiencia en la producción y comercialización de dichos productos y servicios. Además, lograr y mantener competitividad requiere la integración real de las capacidades tecnológicas como pais y como empresas, objetivo al cual deberá propender cualquier esfuerzo en ese sentido.

Es importante entender que hay una relación indisoluble entre innovación y competitividad, para lo cual hay que hacer un esfuerzo que ayude a la adopción de politicas y toma de acciones que incrementen el desarrollo tecnológico de la región en función de la competitividad. Se reconoce que el "conocimiento" por si mismo no transforma las economias o la sociedad, sino que puede hacerlo en el marco de sistemas nacionales $o$ regionales de ciencia, tecnología e innovación, que posibiliten su incorporación al sector productor de bienes y servicios. 
La implementación de modelos y articulación de la innovación como parte del desarrollo productivo, son esenciales a la hora de establecer una nueva empresa. La región andina tiene una capacidad instalada muy baja y se deben aprovechar los sistemas e infraestructura, apoyada con las tecnologías de la información y las comunicaciones y las ideas, que puede derivar en ingreso y políticas sociales en beneficio de la misma sociedad.

La integración en este momento pasa a establecerse como herramienta y vía de cooperación y transferencia de tecnología en función de la movilidad de capitales y la eficiencia vía innovación y competitividad, con miras a la generación de nuevo conocimiento creativo.

La CAN de manera oficial para la innovación propone el siguiente Objetivo según su página oficial (2009):

Promover, estimular y potenciar la innovación tecnológica y su incorporación a los procesos productivos, para coadyuvar en la elevación de las capacidades competitivas de las pequeñas y medianas empresas de diversos sectores, como vía para alcanzar los estándares exigidos por los mercados mundiales y contribuyendo asi, a la superación de la inequidad social y a la creación de bienestar de la sociedad andina en general.

Esto mediante los siguientes aspectos, citados en la página oficial de la CAN (2009):

Promover una mayor integración $e$ interrelación entre los agentes económicos y sociales participantes en los procesos de innovación tecnológica: centros académicos, centros de investigación y desarrollo, empresas, gobiernos nacionales y locales, entes financieros, usuarios y consumidores.

- Apoyar la consolidación de los Sistemas Nacionales de Innovación y conformación del Plan o Programa Andino de Innovación Tecnológica (PAITEC).

- Promover el establecimiento de una visión compartida sobre conceptos y estrategias de estimulo a la innovación, que contribuya a la inserción de la sociedad andina en la "sociedad del conocimiento".

- Realizar estudios que aporten nuevos elementos para la mejor comprensión de los procesos de innovación.

- Determinar los arreglos institucionales de coordinación de los diversos agentes económicos y sociales participantes en esfuerzos de Innovación sobre los Estados Miembros, su nivel de desarrollo, sus potencialidades y los proyectos cientificos y tecnológicos en marcha, asi como el desarrollo de programas de competitividad.

- Establecer un sistema de información y base de datos, que incluya estadísticas, datos macroeconómicos, información institucional $e$ individuos, con el fin de que sirva como base para la investigación $y$ desarrollo de nuevas politicas de innovación y competitividad y conduzca al establecimiento de una Red Institucional de Programas y Centros de Innovación.

- Organizar seminarios, talleres y teleconferencias que propendan a la discusión y profundización de aspectos conceptuales y estratégicos vinculados con la innovación. 
- Identificar oportunidades de cooperación sobre innovación tecnológica.

Estas son las oportunidades que las empresas deben aprovechar y deben implementar modalidades de generación de nuevos productos, innovación y variedad en la oferta interna y exportable.

\section{La Innovación y los procesos regionales de Integración regional}

Según Susana Finquelievich (2007):

Dado que en las últimas décadas la ciencia y la tecnología son, más que nunca, el motor de aceleración del desarrollo y de las transformaciones económicas, la necesidad de promover la innovación, como componente esencial para alimentar a dicho motor, se torna una prioridad politica central (Gurstein, 2003). Por otro lado, el modelo de innovación, si bien está inevitablemente ligado a los proyectos nacionales, no es necesariamente un modelo originado por el Estado central, 'desde arriba hacia abajo', sino que puede ser considerado como basado en las comunidades locales, en su red de actores sociales, o como construido desde abajo hacia arriba. Valenti destaca la necesidad de definir un nuevo marco institucional para la sociedad de la información, caracterizado por dos niveles diferenciados: el nivel macro, constituido por las organizaciones del Estado que establecen las reglas de acción colectiva y el nivel micro.
Este proceso es esencial y los modelos actuales dependen en gran medida de las políticas que se generen desde las organizaciones empresariales y desde los sectores y grupos de investigación, para generar nuevos planteamiento e ideas en función de posibilidades de desarrollo de los países de la subregión andina.

Según Susana Finquelievich (2007)

Gran parte de las inversiones en infraestructura para la innovación se ha focalizado en universidades, centros de investigación, incubadoras de empresas e infraestructuras de tecnología, generalmente allá donde existe suficiente densidad de población, personal calificado y calidad de experiencia en los sistemas existentes como para justificar estas inversiones. Por estas razones, los 'medios innovadores' -la innovación tecnológica basada en el conocimiento, traducida en la producción de bienes $y$ servicios intensivos TIC, o en empresas que actuan en redes organizacionales basadas en las TIC, clusters empresarios, parques de ciencia $y$ tecnología, tecnopolos, etc. - se concentran actualmente en dichas áreas metropolitanas o en sus áreas de influencia, articulados y conectados a través de redes de telecomunicaciones en el conjunto del mundo.

En este sentido, la innovación pasa a ser una de las variables más importantes para la potencialización del aparato productivo y la especialización y aplicación de variables comparativas en desarrollo de la productividad y eficiencia de las empresas.

Por tanto, no son dicotómicas la integración regional y la innovación productiva desde las pequeńas empresas para 
impulsar el desarrollo y la producción de los países.

Las empresas, especialmente las Pymes, requieren de procesos de innovación para la dinamización de la producción de los países, además de apoyarse en la integración regional como soporte en la política exterior y nacional y en el crecimiento de los incentivos para su fomento, vía innovación y generación de nuevo conocimiento.

Según Marleny Cardona \& Carlos Cano (2005):

La actividad innovativa, según Yoguel y Boscherini (1996), se desarrolló en un ambiente económico con diversos tipos de incertidumbres, que son codificadas de distinta forma por las firmas en función de su cultura organizacional y del grado de desarrollo de sus competencias. Estas competencias acumuladas permiten decodificar de distinta forma en conjunto de informaciones y señales del ambiente en términos de conocimientos específicos vinculados a sus particularidades. Este conjunto de códigos, que son desarrollados en forma desigual por las firmas, condiciona la calificación de los recursos humanos presentes en la firma y por lo tanto sus competencias y el grado de complejidad de las innovaciones realizadas.

En este sentido, la actividad productiva pasa de una figura netamente comercial y económica a una estructura de calificación y generación de competencias productivas por parte de las Pymes.

En América andina, especialmente en Colombia, las Pymes se han caracterizado por su accionar limitado y su poco acceso al mercado externo de manera competitiva, dinámica y altamente productiva, especialmente teniendo en cuenta la globalización actual y la interdependencia en un mercado altamente calificado y cualificado para ser generador de crecimiento y productividad.

La transferencia de tecnología para países como Colombia aún se encuentra muy limitada y la aplicación de las tecnologías de la información y las comunicaciones, aún es un tema de ampliación para estas empresas. La segmentación, además, sólo se describe para las grandes empresas generadoras de recursos y mayor volumen concentrado de ingreso al país.

Continúo citando a Marleny Cardona \& Carlos Cano (2005):

La innovación, cuando se desarrolla de forma endógena en una firma o en un territorio, con las competencias especializadas, responde a una lógica de extensión progresiva y de incremento de la experiencia; pero cuando se buscan salidas comerciales y extensiones hacia otros dominios se produce la capacidad de innovación basada en la experiencia acumulada: experiencia cruzada entre productores y experiencia cruzada entre productores y clientes. El limite de este esquema, segun Veltz (1999), es el riesgo de encerrarse en un conjunto limitado de productos y procedimientos.

En ese orden de ideas, la innovación se convierte en un instrumento de ampliación de la producción y la mayor movilidad de capital en función de la competencia entre Estado y entre empresas.

Según Marleny Cardona \& Carlos Cano (2005): 
La actividad innovativa, según Yoguel y Boscherini (1996), se desarrolló en un ambiente económico con diversos tipos de incertidumbres, que son codificadas de distinta forma por las firmas en función de su cultura organizacional y del grado de desarrollo de sus competencias. Estas competencias acumuladas permiten decodificar de distinta forma en conjunto de informaciones y señales del ambiente en términos de conocimientos especificos vinculados a sus particularidades. Este conjunto de códigos, que son desarrollados en forma desigual por las firmas, condiciona la calificación de los recursos humanos presentes en la firma y por lo tanto sus competencias y el grado de complejidad de las innovaciones realizadas.

La capacidad productiva de las Pymes y la innovación para gestión del conocimiento con fines de competencia, capacitación y diversificación de los productos, han limitado mucho a Colombia en el mercado internacional.

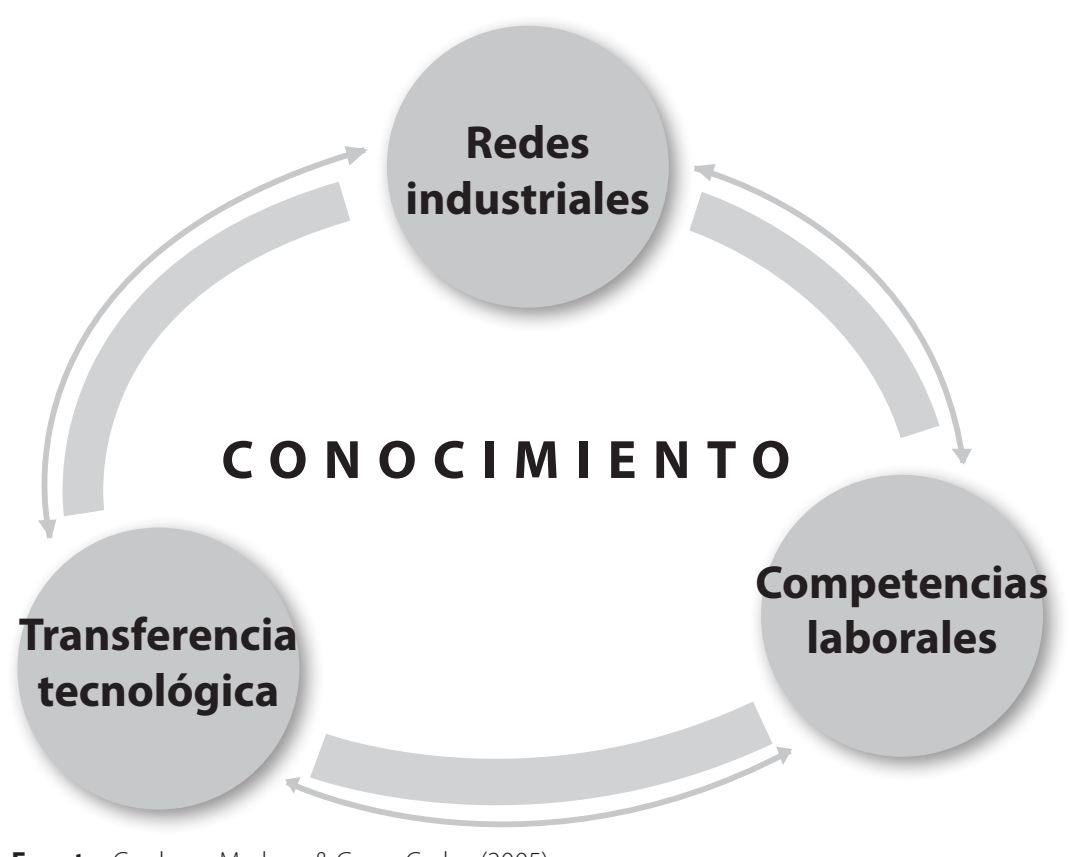

Fuente: Cardona, Marleny \& Cano, Carlos (2005).

Este proceso, vincula la empresas con la generación de conocimiento que hace parte de la dinámica de los países, por lo tanto las Pymes se convertirían en proveedoras de crecimiento y desarrollo para los estados.
Dentro de los lineamientos de la Comunidad Andina, se han estructurado política interna y externa en función de la innovación de la empresa para el servicio del PIB nacional. 
Según el Centro Nacional de Productividad Colombia (2009):

La innovación en las organizaciones está determinada por un cambio importante en los modelos mentales que han servido de referencia para mantener la empresa, pero que no han sido suficientes para crecer $y$ fortalecer su posición en un mercado objetivo. En el mundo de hoy los cambios son más frecuentes particularmente por tres razones: existe mayor competencia, los mercados se han globalizado y las tecnologías se reemplazan más rápido.

Este cambio se ha dado paulatinamente en las empresas Pymes en Colombia, especialmente las concentradas en la capital, Bogotá, pues esta muestra posibilita el accionar exportador y comercial desde el desarrollo productivo.

Esta desigualdad es tan importante de disolver que los países andinos deben posibilitar políticas internas que incrementen el desarrollo sectorial productivo y por lo tanto, el nacional.

Según Esparcia, Noguera \& Ferrer (2003):

Uno de los factores definitorios del desarrollo de las áreas es la capacidad que las empresas de estas regiones tienen para innovar y trasladar las innovaciones (Méndez, R. 2001), ya que si en el territorio no se dan las condiciones idóneas para que las innovaciones se propaguen, se producirá un estancamiento de las empresas y por lo tanto del territorio. Como es lógico, las innovaciones no están todas al mismo nivel, con una gradación bien definida; si bien los procesos de innovación pueden producirse de manera paralela y combinada y no están exentos de una enorme complejidad (Salom, J. et al., 1999):

- En un primer escalón se encuentran las innovaciones que afectan al proceso exclusivamente (más comunes, que se trasladan normalmente a la renovación de maquinaria).

- Por otro lado, tenemos las innovaciones que intentan mejorar los productos mediante la introducción de alguna mejora.

- Por último tenemos las innovaciones que intentan combinar ambas variables y realizan cambios muy profundos, mejorando tanto el producto (que puede ser de nuevo diseño), como los sistemas productivos (sistemas de trabajo, mejora de procesos de marketing, introducción de un sistema de calidad, etc.).

Estos procesos incluyen transferencia tecnológica, como programas de diversificación y acercamiento de la investigación al servicio de la empresa Pyme, para desarrollarla.

En este sentido, hay un instrumento para la generación y perdurabilidad de políticas y apropiación de la tecnología desde lo local a lo regional incluyendo la política exterior y los acuerdos y tratados comunes. Este instrumento es la comunicación y la articulación de políticas macroeconómicas en desarrollo de la innovación de las Pymes, con el fin de establecer de manera común estrategias que sean realmente innovadoras y que beneficien el aparato productivo local y regional, con el fin de reducir la brechas existente entre la periferia y el centro.

Bogotá, al igual que las ciudades que concentran estos procesos, debe establecer 
políticas comunes que deriven en procesos innovadores y generadores de productividad y eficiencia en beneficio del producto interno bruto de la ciudad y del país.

Según Documento oficial de la Unión Europea (2009):

La fase de definición y materialización de las Estrategias Regionales de Innovación, debe complementarse con ejercicios de innovación interregional y transnacional, que permitan el intercambio de experiencias, la definición de las "mejores prácticas" en términos europeos y la cooperación con otras regiones en la ejecución de programas comunes que optimicen los recursos disponibles.

A manera de discusión: los retos de la innovación para los procesos regionales de integración, así como para todos nuestros países que están buscando fuentes de innovación, para la competencia mundial que cada vez es más intensa.

Los factores externos que afectan los procesos económicos de los países, deben estar dirigidos a buscar alternativas de innovación, desde la academia a la empresa pequeña y mediana, para que tengan unas mejores opciones de competir y de hacer que sus empresas sean crecientes y puedan tener ventajas frente al mercado internacional.

Se identifican retos adicionales para los países de América Latina desde el ambiente de la cooperación y la integración regional hacia la innovación en las pequeñas y medianas empresas, se trata de verificar cómo el concepto de innovación puede modificar la estructura productiva de los estados desde sus empresas.

Este reto es tan interesante teniendo en cuenta lo susceptible de las economías de los países en desarrollo y la influencia económica global de los países emergentes en términos de cantidad, que podría semejarse con innovación de los mercados mundiales.

Para la integración regional el tema de la innovación es de gran estudio, en especial para países que subsisten de modos productivos muy manuales y empresas familiares.

El producto de estas empresas desde la innovación está concebido como una adición repetitiva que no alcanza la medición de nuevo y de mejora del producto $y$, por el contrario, se ha encasillado el término de innovación en función del detalle y no en función de lo agregado o transformado.

Es por esto que se debe establecer un proceso de capacitación y continua retroalimentación de lo que en realidad es la innovación y lo que puede realizar el pequeño y mediano empresario, para que este proceso genere los cambios esperados y que estos redunden en el crecimiento y desarrollo de los países de la región.

Por lo tanto, tanto el sector académico como el productivo deben involucrar estrategias de mejoramiento continuo y de visualizar las posibilidades diversas que tiene la innovación desde el producto, en especial para la pequeña y mediana empresa, donde se regule y se genere un sistema productivo dinámico y no estático, continuo, que favorezca la empresa nacional y que el sistema que promueva sea competitivo y productivo eficientemente.

Como se ve en todos los documentos, existen numerosos artículos que hablan de la necesidad de innovar en las empresas y de que este proceso de innovación perdure y no se convierta en una empresa con una idea que sólo dure muy poco. 
Para las Pyme es muy importante generar un esquema de innovación que perdure. Este escenario es muy complejo en la medida que las políticas estatales y la competencia de las grande marcas inmersas en el proceso de globalización han derivado en ajustes necesarios que las empresas deben cumplir, pero que, la mayoría de veces, son costosos y demasiado largos en cuanto a trámites y documentos.

Es por esto importante establecer una política estatal de apoyo que sea efectiva para estas empresas y generar políticas de emprendimiento y apoyo financiero que derive en la sostenibilidad de estas empresas a lo largo del tiempo y bajo las condiciones actuales del mercado.

Este tipo de políticas se deben empezar a estructurar paulatinamente bajo control, de tal forma que las empresas empiecen a fortalecerse y a competir por medio de clúster o en esquemas de productos innovadores con exportaciones a otros países, en los cuales nuestros productos son altamente solicitados.

Actualmente es una oportunidad para Colombia estar en la Secretaría General de la UNASUR (Unión de Naciones del Sur); ojalá este escenario pueda darle visibilidad al pequeño empresario colombiano y apoyarlo para su competitividad a nivel interno y externo. Valiéndonos de estos acuerdos comerciales, donde se fortalezca la industria de los países integrantes de dichos acuerdos, estos escenarios regionales aportarán para el PIB de la región.

Este escenario es enriquecedor en la medida en que se pueden tomar decisiones que apoyen las empresas nacionales y las de los países miembros, generando intercambios comerciales en zonas de libre comercio propicias para la generación de productividad de estas empresas y, por tanto, de estos países.

\section{Referencias}

I. ACS, Zoltan J. \& Audretsch D.B. (1991). R\&D, firm size and innovate activity. En Z.J Acs \& D.B. Audretsch (Eds.), Innovation and Technological Change: An International Comparison. Ann Arbor: University of Michigan Press.

2. Bastos Tigre, P. (1986) Las tendencias internacionales en la electrónica. En Ominami, C (Ed.), La Tercera Revolución Industrial. Buenos Aires: Grupo Editor Latinoamericano.

3. Bernal, Campo E. y Jaramillo, LJ. (1991). La nueva gerencia de proyectos de ciencia y tecnología. Tipologías, roles criticos, productos finales, factores de éxito y evaluación ex-post. (Informe presentado al Centro Internacional de Investigaciones para el Desarrollo). Santafé de Bogotá.

4. Cadena Gómez, G. (1992). Desarrollo tecnológico en el sector cafetero colombiano. (Taller Preparatorio del Simposio del Programa nacional de Desarrollo Tecnológico Industrial y Calidad). Santafé de Bogotá: Colciencias.

5. Cañas, R; Lavados, J \& Marcovitch, J. (1989). Gestión tecnológica y desarrollo. Serie Manuales I\&D. Cinda/Pund/Secab. Santiago de Chile.

6. Castells, P. \& Valls Passola, J. (1997). Tecnología e innovación en la empresa. Dirección y gestión. Barcelona: Edicions Universitat Politécnica de Catalunya.

7. Chaparro, F. (14 de agosto de 1998). Conocimiento, innovación y construcción de sociedad: una agenda para la 
Colombia del Siglo XXI. Santafé de Bogotá.

8. Ciencia, Tecnología y Desarrollo. (1972). El consenso de Brasilia. Sobre la aplicación de la ciencia y la tecnología al desarrollo de América Latina. Secretaría General de la OEA.

9. Ciencia, Tecnología, Desarrollo (1979). Conferencia de las Naciones Unidas sobre la Ciencia y la Tecnología para el Desarrollo. Colciencias. (Julio de 1998). Desafíos en la construcción de una sociedad del conocimiento (Informe de cuatrienio 1995 - 1998). Santafé de Bogotá.

ro.Colciencias. (Septiembre de 1998). Sistema Nacional de Innovación. Nuevos escenarios de la competitividad; Ciencia y Sociedad: Colombia frente al Reto del Tercer Milenio. Santafé de Bogotá.

I I.Colciencias. (Febrero de 1998). Motor de Desarrollo para el País en el Mundo Contemporáneo. Carta de Colciencias. Vol. 21, No 1.

I 2. Colombia. (Octubre-diciembre de 1997). Ciencia \& Tecnología. La Innovación Tecnológica como Factor de Desarrollo. Trece casos exitosos. Vol. 15 No 4.

I 3. Presidencia de la República de Colombia (1994). Al filo de la oportunidad. Misión de Ciencia, Educación y Desarrollo. Consejería para la Modernización del Estado-Colciencias. Bogotá.

I4. Corcoran, E. (1992). Redesigning Research. Scientific American, junio de 1992.

I 5. DNP. (1997). Panorama de la innovación tecnológica en Colombia.

I6. Escardino Benlloch, A. (1995). Reflexiones sobre la $I+D$, la universidad y las empresas Industriales. Universidad Jaime I. Castelló de la Plana.

I7. Espinal Tejada, C. (1997). Centro de Ingeniería Genética y Biotecnología (CIGB). En Gómez Buendía, H. \& Jaramillo,
H. (Compiladores), 37 modos de hacer ciencia en América Latina. Bogotá: Tercer Mundo Editores - Colciencias.

I 8. Freeman, C. (1974). The Economics of Industrial Innovation. England: Penguin Books.

I9. Furnas C.C. \& Mc Carthy Joe. (1975). The Engineer, Life Science Library. Consulting Editors René Dubos, Henry Margenau y C.P Snow. Time- Life Books, New York.

20. Gana, J. (1986). La aparición de nuevos materiales y su impacto sobre el uso de recursos naturales. En Ominami, C (ed.), La Tercera Revolución Industrial. Buenos Aires: RIAL, Grupo Editor Latinoamericano.

21.Gibbons, M.; Limoges, C.; Nowtny, H.; Schwartzman, S.; Scott, P. \& Trow, M. (1997). La nueva producción de conocimiento. La dinámica de la ciencia y la investigación en las sociedades contemporáneas. Barcelona: Ediciones Pomares - Corredor, S.A.

22. Glosario de términos, definiciones y siglas comunes utilizadas en políticas de ciencia y tecnología. Informe Mullin presentado al DNP, Anexo 3. (1996). Basado en Systems of Innovation de Foad Shodjai, Centre for Policy Research on Science and Technology (Cprost), Simon Fraser University (Traducido por Luis Javier Jaramillo).

23. González, MI.; López Cerezo, JA. \& López, J J. (1997). Los Estudios sobre ciencia, tecnología y sociedad. En Rodríguez Alcaraz, J. y otros (eds.), Ciencia, tecnología y sociedad. Contribuciones para una cultura de la paz. Universidad de Granada, Granada.

24. Grupo de Lisboa. (1994). Limites a competiçao. Publicaçoes Europa América. 2a ediçao. Fundaçao Calouste Guilbenkian, Lisboa. 
25.Investigación cientifica y desarrollo tecnológico: una comparación de sus características. (Informe Mullin. Anexo 6. Presentado a la División de Desarrollo Tecnológico del DNP. 1996) (Traducido por Luis Javier Jaramillo).

26.Cardona, M. \& Cano C. (2005). Este artículo hace parte de la investigación: Tecnologias, Organización y Politicas: Mundos de producción de las PyMEs en Colombia en el período 1990-2002, desarrollada el año 2005 por el Grupo de Estudios sectoriales y territoriales -Esyt- de la Universidad EAFIT. En: http://www.banrep.gov.co/documentos/conferencias/medellin/2006/InnovacionEmpresarial.pdf. Consultado 21 de septiembre de 2009.

27.Esparcia, Pérez \& Noguera, Tur Javier Ferrer Sanantonio, Joan Vicente (2003). Boletín de la A.G.E. N.o 36 2003, págs. 149-160. UDERVAL. Departamento de Geografía. Universitat de València. En:http://age.ieg.csic.es/ boletin/36/3610.pdf. Consultado 24 de septiembre de 2009.

28. Méndez, R. (2001). Innovación en la pequeña empresa y redes de cooperación para el desarrollo local. En Martínez Puche, A. (Coord.), El Desarrollo Rural/Local Integrado y el Papel de los Poderes Locales. Universidad de Alicante.

29. Raymond Prada (2009). Página Oficial Centro Nacional de Productividad Colombia. En:http://www.cnp.org.co/ contenidos/Queinnovacionempresarial.php. Consultado 20 de septiembre de 2009.

30.Finquelievich, S. (2007). Universidad de Buenos Aires, Argentina Innovación, tecnología y prácticas sociales en las ciudades: hacia los laboratorios vivientes. Revista CTS, 9(3), 135-152.
3r.Página Oficial Comunidad Andina de Naciones CAN. En www.comunidadandina.org. Consultado 18 de septiembre de 2009.

32. Página Oficial de la UE (España). Programa Sectorial de Competitividad en Innovación empresarial. http://www. ayudaspri.juntaextremadura.net/prianterior/site/pdfs/2plan/anexo\%2013.pdf. Consultado 25 de septiembre de 2009.

33. Grant, R.M. (1997). Dirección Estratégica. Conceptos, Técnicas y Aplicaciones. Madrid: Civitas.

34.Nonaka, I., Takeuchi, H. (1995). The Knowledge-Creating Company. Oxford University Press.

35.OCDE (1992), La Technologie et l'Économie. Les Relations Déterminantes, Programme TEP. OCDE. Paris.

36.OCDE (1993), R\&D Statistics and Output. The Frascati Manuel, OCDE. Paris.

37. OCDE (1997), Manual de OSLO. Paris.

38. Pérez, C. (1996). La modernización industrial en América Latina y la herencia de la substitución de importaciones, Comercio Exterior, 46, 349-63.

39. Rosenberg, N. (1982). Inside the Black Box: Technology and Economics. Cambridge: Cambridge University Press.

40.Rosenberg. N. y Nelson R. (1994). American Universities and technical advance in industry. Research Policy, 23, 323-48.

4I.SECyT (1996). Bases para la discusión de una política de Ciencia y Tecnología, Poder Ejecutivo Nacional, República Argentina.

42.SECyT (2004). Indicadores de Ciencia y Tecnología www.secyt.org, Ministerio de Educación, Ciencia y Tecnología. Secretaría de Ciencia y Tecnología 
e Innovación Productiva, República Argentina. Buenos Aires.

43.SECyT (2005). Plan Nacional de Ciencia Tecnología e Innovación (PNCTIP), Secretaría de Ciencia y Tecnología e Innovación Productiva.

44. Stoneman, P. (1995. Introduction. En S. Paul (Ed.), Handbook of the Economics and Technological Change. Blackwell. Oxford, Cambridge.

45. Teece, D. (2000). Firm Capabilities and Economic Development: Implications for Newly Industrializing Economies. Berkley: University of California. En Kim L. y Nelson R. 2000) Tecnology, learning and Innovation, London: Cambridge University Press (Eds.)

46.Porter, M. (1987). Ventaja Competitiva. CECSA: México.

47.Prusak, L. (1996). The Knowledge Advantage. Strategy \& Leadership. March/ April. P. 6-8.

48. Roos, J., Roos, G., Edvinsson \& L., Dragonetti, N.C., (1997) Intellectual Capital. London: Macmillan Business.
49. Sveiby, K. E. (1997). The New Organizational Wealth. USA: Berett-Koehler Publishers, Inc.

50.Viedma, J.M. (2001). IICBS Innovation Intellectual Capital Benchmarking System. World Congress on Intellectual Capital Readings. Butterworth Heinemann. Edited by Nick Bontis. P.243-265.

5 I. Viedma, J.M. (2000). "Gestión del Conocimiento y del capital intelectual". Nueva Empresa.com No454/octubre 2000 (Año XXII) Suplemento: Cuadernos de Management para una dirección eficaz. pp. 99-105.

52.Yoguel, G.; Novick, M.; Milesi, D.; Roitter, S. \& Borello, J. (2004). Información y conocimiento: la difusión de las tecnologías de información y comunicación en la industria manufacturera Argentina. Revista Cepal, No. 82. 